\title{
Oversampling in Shift-Invariant Spaces With a Rational Sampling Period
}

\author{
A. G. García, M. A. Hernández-Medina, and G. Pérez-Villalón
}

\begin{abstract}
It is well known that, under appropriate hypotheses, a sampling formula allows us to recover any function in a principal shift-invariant space from its samples taken with sampling period one. Whenever the generator of the shift-invariant space satisfies the Strang-Fix conditions of order $r$, this formula also provides an approximation scheme of order $r$ valid for smooth functions. In this paper we obtain sampling formulas sharing the same features by using a rational sampling period less than one. With the use of this oversampling technique, there is not one but an infinite number of sampling formulas. Whenever the generator has compact support, among these formulas it is possible to find one whose associated reconstruction functions have also compact support.
\end{abstract}

Index Terms-Approximation order, oversampling, sampling in shift-invariant spaces, shift-invariant spaces.

\section{INTRODUCTION}

$\mathbf{T}$ HE sampling theory in shift-invariant spaces, in particular in wavelets subspaces, has been largely studied in the last few years. As pointed out by Unser in [16], an appropriate choice for the generator $\varphi$ (for instance, a B-spline) eliminates some of the problems associated with the classical Shannon's sampling theory; in particular, those related to the slow decay of the sinc function. Thus, we consider a shift-invariant space

$$
V_{\varphi}^{2}:=\left\{\sum_{n \in \mathbb{Z}} a[n] \varphi(t-n):\{a[n]\}_{n \in \mathbb{Z}} \in \ell^{2}(\mathbb{Z})\right\} \subset L^{2}(\mathbb{R})
$$

where the function $\varphi \in L^{2}(\mathbb{R})$ is a stable generator, i.e., the sequence $\{\varphi(\cdot-n)\}_{n \in \mathbb{Z}}$ is a Riesz basis for $V_{\varphi}^{2}$. The starting point of the sampling theory in $V_{\varphi}^{2}$ (see [1] and [17]) is that, under appropriate hypotheses, any function $f \in V_{\varphi}^{2}$ can be recovered from a sequence of samples taken with sampling period $T_{\mathrm{s}}=1$, by means of the sampling formula

$$
f(t)=\sum_{n \in \mathbb{Z}} f(n) S(t-n), \quad t \in \mathbb{R} .
$$

Manuscript received October 28, 2008; accepted March 23, 2009. First published April 21, 2009; current version published August 12, 2009. The associate editor coordinating the review of this manuscript and approving it for publication was Prof. Pierre Vandergheynst. This work has been supported by the Grant MTM2009-08345 from the D.G.I. of the Spanish Ministerio de Ciencia y Tecnología.

A. G. García is with the Departamento de Matemáticas, Universidad Carlos III de Madrid, 28911 Leganés-Madrid, Spain (e-mail: agarcia@math.uc3m.es).

M. A. Hernández-Medina is with the Departamento de Matemática Aplicada, E.T.S.I.T., Universidad Politécnica de Madrid, 28040 Madrid, Spain (e-mail: mahm@mat.upm.es).

G. Pérez-Villalón is with the Departamento de Matemática Aplicada a la Telecomunicación, E.T.U.I.T., Universidad Politécnica de Madrid, 28031 Madrid, Spain (e-mail: gperez@euitt.upm.es).

Digital Object Identifier 10.1109/TSP.2009.2021497
The involved reconstruction function $S$ is given by

$$
S(t)=\sum_{n \in \mathbb{Z}} c[n] \varphi(t-n)
$$

where $\{c[n]\}_{n \in \mathbb{Z}}$ is the sequence whose $z$-transform is $C(z)=$ $\left[\sum_{n \in \mathbb{Z}} \varphi(n) z^{-n}\right]^{-1}$ which is assumed to be stable, i.e., there exist two positive constants $A, B$ such that $0<A \leq|C(z)| \leq$ $B<\infty$ a.e. on $|z|=1$. In other words, any $f \in V_{\varphi}^{2}$ can be recovered from the discrete prefiltering of its samples: $a[n]=$ $\sum_{k} f(k) c[n-k]$, by $f(t)=\sum_{n} a[n] \varphi(t-n)$.

Due to the good approximation properties from shift-invariant spaces (see [4], [11], and [14]), the scaling of the sampling formula (1) allows us to approximate regular functions from a sequence of samples taken with a small sampling period $h>0$. Specifically, if the generator $\varphi$ satisfies the Strang-Fix conditions of order $r$ [see infra (12)] then, any function $f$ in the Sobolev space $W_{\infty}^{r}(\mathbb{R}):=\left\{f:\left\|D^{n} f\right\|_{\infty}<\infty, n \leq r\right\}$ satisfies

$$
\left|f(t)-\sum_{n \in \mathbb{Z}} f(n h) S\left(\frac{t}{h}-n\right)\right| \leq K\left\|D^{r} f\right\|_{\infty} h^{r}, \quad t \in \mathbb{R}
$$

where the constant $K$ does not depend on $f$ and $h$ (see [9]-[11]), and $D^{r}$ denotes the derivative operator of order $r$.

Whenever $C(z)$ is not an FIR filter, which almost always happens (see [8]), the reconstruction function $S$ does not have compact support, even when the generator has it. A reconstruction function $S$ with compact support implies low computational complexity and avoids truncation errors. In the case of sequence $\{c[n]\}_{n \in \mathbb{Z}}$ in (2) being finite, the reconstruction function $S$ could inherit most of the good properties of the generator $\varphi$. In particular, the function $S$ would have compact support if $\varphi$ does. Unfortunately, the coefficients $\{c[n]\}_{n \in \mathbb{Z}}$ form, in general, an infinite sequence. An important exception occurs when the generator $\varphi$ is the B-spline of degree 1 where the Strang-Fix conditions of order 2 are satisfied.

The approximation in (3) is an interpolation scheme, i.e., $f(n h)=\sum_{k} f(k h) S(n-k)$. Another possibility is to use a quasi-interpolation scheme, where the above interpolation condition holds for polynomials of degree $\leq r$. This quasi-interpolation technique allows us to get suitable FIR filters for the aforementioned sampling problem (see [3]).

In this work we study the recovery of functions in $V_{\varphi}^{2}$ from their samples taken with a rational sampling period $T_{\mathrm{s}}=p / q \leq 1$, instead of $T_{\mathrm{s}}=1$, and their related approximation schemes. By using this oversampling technique, many infinite sampling formulas do exist and some of them involve $q$ 
compactly supported reconstruction functions $S_{0}, S_{1}, \ldots, S_{q-1}$ instead of only one. In other words, it entails an FIR filter-bank, instead of an IIR filter. This could be suitable, specially, when the involved filters have small support.

The paper is organized as follows. In Section II, we obtain the sampling formulas valid for a shift-invariant space larger that $V_{\varphi}^{2}$ by using samples taken at a rational sampling period less than one. We also prove that these sampling formulas satisfy an approximation property as in (3), whenever $\varphi$ satisfies the Strang-Fix conditions of some fixed order. Section III is devoted to the existence and computation of reconstruction functions with compact support in the particular case of $T_{\mathrm{s}}=p /(p+1)$. Finally, an Appendix includes the technical proofs of the results given in Sections II and III.

\section{SAmpling Formulas With Rational SAmpling Period}

In order to obtain an approximation result like (3) which involves the $L^{\infty}$-norm, we first deduce sampling formulas valid for a subspace of $L^{\infty}(\mathbb{R})$ larger than $V_{\varphi}^{2}$. Indeed, some extra conditions on the stable generator $\varphi$ in $L^{2}(\mathbb{R})$ allows us to work in the shift-invariant space

$$
V_{\varphi}^{\infty}:=\left\{\sum_{n \in \mathbb{Z}} a[n] \varphi(t-n): \lim _{|n| \rightarrow \infty} a[n]=0\right\} .
$$

Specifically, throughout this paper we assume that the stable generator $\varphi$ is a continuous function on $\mathbb{R}$ having some decay property. In fact, we assume that $\varphi$ belongs to the Wiener space $W\left(L^{\infty}, \ell^{1}\right)$. Recall that

$$
W\left(L^{\infty}, \ell^{1}\right):=\left\{f: \sum_{n \in \mathbb{Z}}\left\|f \chi_{[n, n+1)}\right\|_{\infty}<\infty\right\} .
$$

Note that $W\left(L^{\infty}, \ell^{1}\right) \subset L^{1}(\mathbb{R}) \cap L^{2}(\mathbb{R}) \cap L^{\infty}(\mathbb{R})$ (see [5]). Observe that $\varphi \in W\left(L^{\infty}, \ell^{1}\right) \cap C(\mathbb{R})$ implies $\sup _{t \in[0,1)} \sum_{n \in \mathbb{Z}}|\varphi(t+n)|<\infty$ and $\lim _{|t| \rightarrow \infty} \varphi(t)=0$. Thus, the space $V_{\varphi}^{\infty}$ coincides with the closed subspace in $L^{\infty}(\mathbb{R})$ generated by the integer shifts of $\varphi$ (see [11]), and it is a space of continuous functions [10]. Observe that any generator satisfying $|\varphi(t)| \leq C\left(1+|t|^{1+\delta}\right)^{-1}$ for some $C>0$ and $\delta>0$ belongs to $W\left(L^{\infty}, \ell^{1}\right)$; in particular, any compactly supported generator $\varphi$. We also assume that the sampling period $T_{\mathrm{s}}$ is a rational sampling period less than or equal to $1: T_{\mathrm{s}}:=p / q \leq 1$, $p, q \in \mathbb{N}$.

We split the theoretical discussion into two separate subsections.

\section{A. The Perfect Reconstruction Process}

In order to introduce some notation and the main ideas in the present work, let us first consider the recovery of a function $f$ in the linear span of the sequence $\{\varphi(\cdot-n)\}_{n \in \mathbb{Z}}$, i.e., a function $f$ of the form $f(t)=\sum_{n \in \mathbb{Z}} a[n] \varphi(t-n)$ where $\{a[n]\}$ is a finite sequence. Its samples taken at the points $\left\{m T_{\mathrm{s}}\right\}_{m \in \mathbb{Z}}$ admit a simple expression in terms of the sequence $\{a[n]\}_{n \in \mathbb{Z}}$. Specifically and, for $j=0,1, \ldots, q-1$, we have

$$
\begin{aligned}
c_{j}[n] & :=f\left(n p+j T_{\mathrm{s}}\right)=\sum_{m \in \mathbb{Z}} a[m] \varphi\left(n p+j T_{\mathrm{S}}-m\right) \\
& =\sum_{k=0}^{p-1} \sum_{m \in \mathbb{Z}} a[-k+m p] \varphi\left(p n+j T_{\mathrm{S}}-p m+k\right) \\
& =\sum_{k=0}^{p-1}\left(a_{k} * \varphi_{j, k}\right)[n]
\end{aligned}
$$

where $a_{k}=\left\{a_{k}[n]:=a[-k+n p]\right\}_{n \in \mathbb{Z}}, \varphi_{j, k}=\left\{\varphi_{j, k}[n]:=\right.$ $\left.\varphi\left(p n+j T_{\mathrm{s}}+k\right)\right\}_{n \in \mathbb{Z}}$ and $*$ denotes the usual convolution operator. Computing the $\mathrm{z}$-transform in the above equality (notice that $\varphi_{j, k} \in \ell^{1}(\mathbb{Z})$ ), we obtain that

$$
C_{j}(z)=\sum_{k=0}^{p-1} H_{j, k}(z) A_{k}(z), \quad j=0,1, \ldots, q-1
$$

where $H_{j, k}(z), A_{k}(z)$, and $C_{j}(z)$ are the $z$-transforms of $\varphi_{j, k}$, $a_{k}$, and $c_{j}$, respectively. The matrix form of equations in (4) reads as

$$
\begin{aligned}
& {\left[C_{0}(z) C_{1}(z) \cdots C_{q-1}(z)\right]^{\top}} \\
& =H(z)\left[A_{0}(z) A_{1}(z) \cdots A_{p-1}(z)\right]^{\top}
\end{aligned}
$$

where $H(z)$ is the $q \times p$ matrix defined by

$$
\begin{aligned}
H(z) & :=\left[H_{j, k}\right]_{\substack{j=0,1, \ldots, q-1 \\
k=0,1, \ldots, p-1}} \\
& =\left[\sum_{n \in \mathbb{Z}} \varphi\left(j T_{\mathrm{s}}+k+p n\right) z^{-n}\right]_{\substack{j=0,1, \ldots, q-1 \\
k=0,1, \ldots, p-1}}
\end{aligned} .
$$

The reader who is familiar with the filter-bank theory can observe that the matrix $H(z)$ coincides with the polyphase matrix of a related filter-bank. The important relationship between the filter-banks theory and sampling in shift-invariant spaces was established in [8] and [15].

Equality (5) shows the important role of the left inverse matrices (if any) of $H(z)$ for the problem that we are dealing with. Any left inverse of the matrix $H(z)$, defined in $|z|=1$, allows us to recover the functions $A_{0}(z), A_{1}(z), \ldots, A_{p-1}(z)$; as a consequence, any function $f \in \operatorname{span}\{\varphi(\cdot-n)\}_{n \in \mathbb{Z}}$ can be recovered from its samples. However, as we are mainly interested in left inverses of $H(z)$ leading to a result like (3), we only consider those whose entries belong to the class

$$
\mathcal{A}:=\left\{\sum_{n \in \mathbb{Z}} a[n] z^{-n}:\{a[n]\}_{n \in \mathbb{Z}} \in \ell^{1}(\mathbb{Z})\right\} .
$$

The next lemma gives a necessary and sufficient condition for the existence of such left inverses. As usual, we denote by $H^{*}(z)$ the transpose conjugate of the matrix $H(z)$.

Lemma 1: Assume that $\varphi$ is a continuous generator in $W\left(L^{\infty}, \ell^{1}\right)$, and let $H(z)$ be the $q \times p$ matrix defined in (6). There exists a $p \times q$ matrix $G(z)$ with entries in $\mathcal{A}$ and satisfying

$$
G(z) H(z)=\mathbb{1}_{p}, \quad|z|=1
$$


if and only if $H(z)$ has full rank on $|z|=1$, i.e., rank $H(z)=p$ on $|z|=1$. If these equivalent conditions hold, one of these left inverses is the pseudo-inverse $H^{\dagger}(z):=\left[H^{*}(z) H(z)\right]^{-1} H^{*}(z)$. Any other left inverse $G(z)$ is given by

$$
G(z)=H^{\dagger}(z)+U(z)\left[\rrbracket_{q}-H(z) H^{\dagger}(z)\right]
$$

where $U(z)$ is any $p \times q$ matrix with entries in $\mathcal{A}$.

See the proof in the Appendix.

Notice that if $q=p$ there exists a unique left inverse $G(z)$ but if $q>p$ there are many left inverses. Associated with each of these left inverses

$$
G(z)=\left[G_{k, j}(z)\right]_{\substack{k=0,1, \ldots, p-1 \\ j=0,1, \ldots q-1}}
$$

we consider $q$ reconstruction functions $S_{j}^{G}, j=0,1, \ldots, q-1$, defined in the following way: As $G_{k, j}(z) \in \mathcal{A}$ we can express $G_{k, j}(z)$ in a unique way as

$$
G_{k, j}(z)=\sum_{n \in \mathbb{Z}} g_{k, j}[n] z^{-n}
$$

where $g_{j, k} \in \ell^{1}(\mathbb{Z})$. For $j=0,1, \ldots, q-1$, let $g_{j}=\left\{g_{j}[n]\right\}_{n \in \mathbb{Z}}$ be the sequence defined by $g_{j}[n]:=g_{k, j}[m]$, when $n=-k+m p, k=0,1, \ldots, p-1, m, n \in \mathbb{Z}$. In other words, the sequences $g_{j}, j=0,1, \ldots, q-1$, are those such that

$$
G(z)=\left[\sum_{m \in \mathbb{Z}} g_{j}[-k+p m] z^{-m}\right]_{\substack{k=0,1, \ldots, p-1 \\ j=0,1, \ldots, q-1}} .
$$

Next, consider the functions

$$
S_{j}^{G}(t):=\sum_{n \in \mathbb{Z}} g_{j}[n] \varphi(t-n), \quad j=0,1, \ldots, q-1 .
$$

The following theorem gives, for each one of the aforesaid left inverse matrices $G(z)$, a sampling formula which allows us to recover, from its samples taken at the points $\left\{m T_{\mathrm{s}}\right\}_{m \in \mathbb{Z}}$, any function in $V_{\varphi}^{\infty}$.

Theorem 1: Assume that the generator $\varphi$ is continuous on $\mathbb{R}$ and belongs to $W\left(L^{\infty}, \ell^{1}\right)$. Let $G(z)$ be a $p \times q$ matrix with entries in $\mathcal{A}$ and satisfying (7). Then, for any $f \in V_{\varphi}^{\infty}$

$$
f(t)=\sum_{j=0}^{q-1} \sum_{n \in \mathbb{Z}} f\left(j T_{\mathrm{s}}+p n\right) S_{j}^{G}(t-p n), \quad t \in \mathbb{R}
$$

where the sampling functions $S_{j}^{G}, j=0,1, \ldots, q-1$, are given by (8). The series in (9) converges absolutely and uniformly on $\mathbb{R}$.

See the proof in the Appendix . Notice that formula (9) can be understood as follows: After the discrete prefiltering of the samples of $f$ via a filter-bank [see (8)]

$$
a[n]=\sum_{j=0}^{q-1} \sum_{k} f\left(j T_{\mathrm{s}}+p k\right) g_{j}(n-p k)
$$

we recover $f$ by $f(t)=\sum_{n} a[n] \varphi(t-n)$.

\section{B. The Approximation Scheme}

Recall that we have assumed that $\varphi$ is a stable generator for $L^{2}(\mathbb{R})$; equivalently, there exist two constants $A, B>0$ such that $0<A \leq \sum_{n \in \mathbb{Z}}|\widehat{\varphi}(w+n)|^{2} \leq B<\infty$, where $\widehat{\varphi}(\xi):=$ $\int_{-\infty}^{\infty} \varphi(t) e^{-2 \pi i \xi t} d t$ denotes the Fourier transform of $\varphi$. The orthogonal projector of $L^{2}(\mathbb{R})$ onto $V_{\varphi}^{2}$ is given by

$$
(P f)(t):=\sum_{n \in \mathbb{Z}}\left\langle f, \varphi_{d}(\cdot-n)\right\rangle \varphi(t-n)
$$

where the dual function $\varphi_{d}$ is given by

$$
\widehat{\varphi}_{d}(w):=\frac{\widehat{\varphi}(w)}{\sum_{n \in \mathbb{Z}}|\widehat{\varphi}(w+n)|^{2}} .
$$

Since the sequence $\{\varphi(\cdot-n)\}_{n \in \mathbb{Z}}$ is a Riesz sequence for $L^{2}(\mathbb{R})$, the operator $P$, defined by (10), is also a projector of $L^{\infty}(\mathbb{R})$ onto $V_{\varphi}^{\infty}$ (see [11, Theorem 5.2]).

In what follows we need some further decay for the generator $\varphi$. For a fixed $r \in \mathbb{N}$, assume that the function $\varphi(t)(1+|t|)^{r} \in$ $W\left(L^{\infty}, \ell^{1}\right)$. Hence, the generator decay fast enough, so that the derivatives $D^{n} \widehat{\varphi}$ exist and are continuous functions for $n<r$. If the generator $\varphi$ satisfies the Strang-Fix conditions of order $r$, i.e.,

$$
\widehat{\varphi}(0) \neq 0, \quad D^{n} \widehat{\varphi}(m)=0, \quad n<r, \quad m \in \mathbb{Z} \backslash\{0\}
$$

then the operator $P$ provides approximation order $r$ in the Sobolev space $W_{\infty}^{r}(\mathbb{R})$. Specifically (see [11, Theorem 5.2]), for any $f \in W_{\infty}^{r}(\mathbb{R})$ we have

$$
\left\|f-\sigma_{h} P \sigma_{1 / h} f\right\|_{\infty} \leq K\left\|D^{r} f\right\|_{\infty} h^{r}
$$

where $\sigma_{h} f:=f(\cdot / h)$, and $K$ is a constant independent of $f$ and $h>0$. In next theorem we deduce, from this result, that the sampling formula (9) also provides approximation order $r$ in the Sobolev space $W_{\infty}^{r}(\mathbb{R})$. Thus, any function in $W_{\infty}^{r}(\mathbb{R})$ can be approximated from its samples at $\{n h\}_{n \in \mathbb{Z}}$ taking $h>0$ small enough. The validity of this approximation technique for the signal processing community was pointed out by Unser and Daubechies in [14]. See also the subsequent [3].

Theorem 2: Assume that $\varphi$ is a continuous stable generator such that $\varphi(t)(1+|t|)^{r} \in W\left(L^{\infty}, \ell^{1}\right)$. Let $G(z)$ be a $p \times q$ matrix with entries in $\mathcal{A}$ and satisfying (7). If the generator $\varphi$ satisfies the Strang-Fix conditions of order $r$ then, for any $f \in$ $W_{\infty}^{r}(\mathbb{R}) \cap C(\mathbb{R})$, we have

$$
\begin{array}{r}
\left\|f(t)-\sum_{j=0}^{q-1} \sum_{n \in \mathbb{Z}} f\left(j T_{\mathrm{s}} h+p n h\right) S_{j}^{G}\left(\frac{t}{h}-p n\right)\right\|_{\infty} \\
\leq K\left\|D^{r} f\right\|_{\infty} h^{r}
\end{array}
$$

where the reconstruction functions $S_{j}^{G}, j=0,1, \ldots, q-1$, are given by (8) and $K$ is a constant independent of $f$ and $h$.

See the proof in the Appendix .

Notice that the B-spline of degree $n>0$ satisfies the hypotheses in the theorem for $r=n+1$. 


\section{COMPACTLY SUPPORTED RECONSTRUCTION FUNCTIONS $S_{j}^{G}$}

In this section, we assume that the generator $\varphi$ has compact support. Then the entries of the matrix $H(z)$ are Laurent polynomials. If the matrix $H(z)$ has a left inverse $G(z)=\left[\sum_{n \in \mathbb{Z}} g_{j}[-k+p n] z^{-n}\right]_{\substack{k=0,1, \ldots, p-1 \\ j=0,1, \ldots, q-1}}$ whose entries are also Laurent polynomials, then the sum in (8) giving the corresponding reconstruction functions $S_{j}^{G}(t), j=0,1, \ldots, q-1$, will be finite. Therefore, the reconstruction functions can be easily calculated and they inherit most of the good features of the generator $\varphi$; in particular, they will have compact support.

In [12, Theorem 1] there is a necessary and sufficient condition for the existence of a polynomial left inverse of a given polynomial matrix. The same proof, with minor modifications, applies when we consider Laurent polynomial, obtaining the following theorem.

Theorem 3: Let $p \leq q$. A $q \times p$ matrix $H(z)$ whose entries are Laurent polynomials has a left inverse whose entries are also Laurent polynomials if and only if $\operatorname{rank} H(z)=p$ for all $z \neq 0$ or, equivalently, if the greatest common divisor of the $p \times p$ minors of $H(z)$ is a monomial.

The proof of this theorem is constructive, i.e., it gives a polynomial left inverse matrix involving, in general, a high degree. See [12] and the references therein for other methods to compute a polynomial left inverse matrix. Next, we give an effective method for the important case of minimum oversampling rate.

\section{A. The Case of the Sampling Period $T_{\mathrm{s}}=p /(p+1)$ for $p \geq 1$}

In order to introduce the lowest oversampling rate it is advisable to take the sampling period $T_{\mathrm{s}}=p /(p+1)$, i.e., $q=p+1$ for $p \geq 1$. Without loss of generality we can assume that

$$
\operatorname{supp} \varphi \subseteq[0, R]
$$

for some $R>0$. Indeed, if $\operatorname{supp} \varphi \subseteq[a, b]$ then we could consider the generator $\varphi_{1}(t):=\varphi(t+[a])$, where $[a]$ denotes the integral part of $a$; it is evident that $V_{\varphi_{1}}^{\infty}=V_{\varphi}^{\infty}$ and $\operatorname{supp} \varphi_{1} \subseteq$ $[0, b-[a]]$.

If we take $p \geq R$, then the $(p+1) \times p$ matrix $H(z)$ has a simple expression. Namely, $H(z)=B z+A$ where the $(p+$ 1) $\times p$ matrices $A$ and $B$ given by

$$
\begin{aligned}
& A=[\varphi(j T+k)]_{\substack{j=0,1, \ldots, p \\
k=0,1, \ldots, p-1,}}
\end{aligned}
$$

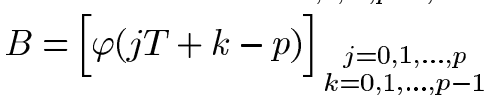

satisfy $A_{j, k}=0$, whenever $p j /(p+1)+k \geq R, A_{0,0}=0$, and $B_{j, k}=0$, whenever $j+k<p+1$ or $p j /(p+1)+k \geq p+R$, i.e., they look like

$$
A=\left[\begin{array}{cccccccc}
0 & * & \cdots & * & * & 0 & \cdots & 0 \\
* & * & \cdots & * & * & 0 & \cdots & 0 \\
* & * & \cdots & * & 0 & 0 & \cdots & 0 \\
\vdots & \vdots & . & \vdots & \vdots & \vdots & & \vdots \\
* & * & \cdots & 0 & 0 & 0 & \cdots & 0 \\
* & 0 & \cdots & 0 & 0 & 0 & \cdots & 0 \\
0 & 0 & \cdots & 0 & 0 & 0 & \cdots & 0 \\
\vdots & \vdots & & \vdots & \vdots & \vdots & & \vdots \\
0 & 0 & \cdots & 0 & 0 & 0 & \cdots & 0
\end{array}\right]
$$

and

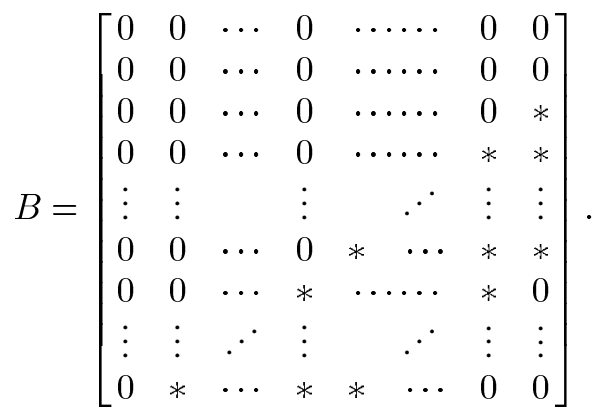

We are concerned in the computation of a polynomial left inverse (if any) of the polynomial matrix $H(z)$. Whenever $p=1$ or $p=2$, it is straightforward to obtain, under mild conditions, an scalar left inverse of $H(z)$.

Next we deal with the general case $p \geq 3$. To this end, consider the square matrix $\mathbf{M} \in \mathbb{C}^{\left(p^{2}-p-1\right) \times\left(p^{2}-p-1\right)}$ defined by

$$
\mathbf{M}:=\left[\begin{array}{ccccc}
\widetilde{A} & & & & \\
\widetilde{B} & A & & & \\
& B & A & & \\
& & \ddots & \ddots & \\
& & & B & A
\end{array}\right]
$$

where the block $A$ is repeated $p-2$ times, $\widetilde{A}$ is the first row of $A$ without the first entry $A_{0,0}=0$ and, $\widetilde{B}$ is the $(p+1) \times(p-1)$ matrix obtained by deleting the first column in $B$, i.e., $\widetilde{A}=$ $[\varphi(k)]_{k=1, \ldots, p-1}$ and $\widetilde{B}=[\varphi(j T+k-p)]_{k=1, \ldots, p-1}^{j=0, \ldots, p}$.

Assuming that $\mathbf{M}$ is a nonsingular matrix in $\mathbb{C}^{\left(p^{2}-p-1\right) \times\left(p^{2}-p-1\right)}$ we obtain a polynomial left inverse of the matrix $H(z)$ of degree $p-2$ as follows: Consider the $p \times\left(p^{2}-p-1\right)$ matrix $\mathbf{N}$ formed with the last $p$ rows of the inverse matrix $\mathbf{M}^{-1}$ partitioned its columns as follows:

$$
\mathbf{N}:=\left[X^{p-2}\left|X^{p-3}\right| \ldots\left|X^{1}\right| X^{0}\right]
$$

where

$$
\begin{aligned}
X^{p-2} & =\left[X_{k, 0}^{p-2}\right]_{k=0,1, \ldots, p-1} \in \mathbb{C}^{p \times 1} \\
X^{l} & =\left[X_{k, j}^{l}\right]_{\substack{k=0,1, \ldots, p-1 \\
j=0,1, \ldots, p}} \in \mathbb{C}^{p \times(p+1)}, \quad l=0,1, \ldots, p-3 .
\end{aligned}
$$

Obviously, it satisfies

$$
\left[X^{p-2}\left|X^{p-3}\right| \cdots\left|X^{1}\right| X^{0}\right] \mathbf{M}=\left[\mathbb{O}_{p, p^{2}-2 p-1} \mid \mathbb{Q}_{p}\right]
$$

where $\mathbb{O}_{p, q}$ denotes the $p \times q$ zero matrix. That is,

$$
\begin{aligned}
& X^{p-2} \widetilde{A}+X^{p-3} \widetilde{B}=\mathbb{O}_{p, p-1} \quad X^{p-3} A+X^{p-4} B=\mathbb{O}_{p, p} \\
& X^{p-4} A+X^{p-5} B=\mathbb{O}_{p, p} \quad X^{p-5} A+X^{p-6} B=\mathbb{O}_{p, p} \\
& X^{1} A+X^{0} B=\mathbb{O}_{p, p} \quad X^{0} A=\mathbb{1}_{p} .
\end{aligned}
$$

Now, we prove that

$$
G(z):=\left[X^{p-2} \mid \mathbb{O}_{p, p}\right] z^{p-2}+X^{p-3} z^{p-3}+\cdots+X^{1} z+X^{0}
$$


is a polynomial left inverse of the matrix $H(z)=B z+A$. Indeed

$$
\begin{aligned}
\left(\left[X^{p-2} \mid \mathbb{O}_{p, p}\right] z^{p-2}+X^{p-3} z^{p-3}+\cdots+X^{0}\right) \\
(B z+A) \\
=\left[X^{p-2} \mid \mathbb{O}_{p, p}\right] B z^{p-1} \\
\quad+\left\{\left[X^{p-2} \mid \mathbb{O}_{p, p}\right] A+X^{p-3} B\right\} z^{p-2} \\
\quad+\left(X^{p-3} A+X^{p-4} B\right) z^{p-3} \\
\quad+\cdots+\left(X^{1} A+X^{0} B\right) z+X^{0} A .
\end{aligned}
$$

Having in mind (18), that $\left[X^{p-2} \mid \mathbb{O}_{p, p}\right] B=\mathbb{O}_{p, p}$ since the first row of $B$ is null, and that

$$
\begin{aligned}
{\left[X^{p-2} \mid \mathbb{O}_{p, p}\right] A+} & X^{p-3} B \\
& =\left[\mathbb{O}_{p, 1} \mid X^{p-2} \widetilde{A}\right]+\left[\mathbb{O}_{p, 1} \mid X^{p-3} \widetilde{B}\right]=\mathbb{O}_{p, p}
\end{aligned}
$$

since $A_{0,0}=0$ and the first column of $B$ is null, we conclude that $G(z) H(z)=\mathbb{1}_{p}$.

Using that $G(z)=\left[\sum_{n \in \mathbb{Z}} g_{j}[-k+p n]^{-n}\right]_{\substack{k=0,1, \ldots, p-1 \\ j=0,1, \ldots, p}}$, we obtain that the reconstruction functions corresponding to this left inverse are

$$
\begin{aligned}
S_{j}^{G}(t) & :=\sum_{l=0}^{p-3} \sum_{k=0}^{p-1} X_{k, j}^{l} \varphi(t+l p+k), \quad j=1,2, \ldots, p, \\
S_{0}^{G}(t) & :=\sum_{l=0}^{p-2} \sum_{k=0}^{p-1} X_{k, 0}^{l} \varphi(t+l p+k) .
\end{aligned}
$$

Notice that, for $j=1,2, \ldots, p$, the reconstruction function $S_{j}^{G}$ is a linear combination of $p^{2}-2 p$ shifts of the generator $\varphi$ and it has its support in the interval $\left[-p^{2}+2 p+1, R\right]$, while $S_{0}^{G}$ is supported in the interval $\left[-p^{2}+p+1, R\right]$. The above procedure can be gathered as a theorem.

Theorem 4: Assume that $\operatorname{supp} \varphi \subseteq[0, R]$ and take $p \in \mathbb{N}$ such that $p \geq R$. For the sampling period $T_{\mathrm{s}}=p /(p+1)$ the associated $(p+1) \times p$ polyphase matrix $H(z)$ in (6) can be written as $H(z)=B z+A$ where the $(p+1) \times p$ scalar matrices $A$ and $B$ are described in (15)-(16). If the $\left(p^{2}-p-1\right) \times\left(p^{2}-p-1\right)$ matrix $\mathbf{M}$ in (17) is nonsingular, then the matrix $H(z)$ possess a polynomial left inverse $G(z)$ given in (19) from which we obtain the compactly supported reconstruction functions $S_{j}^{G}$, $j=0,1, \ldots, p$, given in (20).

Finally, it is worth mentioning that work in progress allows us to guess that reconstruction functions (20) have minimal support when $p$ is the smallest natural number $p \geq R$.

\section{B. A Toy Example Involving the Quadratic B-Spline}

Consider the generator $\varphi(t):=N_{3}(t)$, the quadratic B-spline

$$
N_{3}(t):= \begin{cases}\frac{t^{2}}{2} & t \in[0,1) \\ \frac{6 t-2 t^{2}-3}{2} & t \in[1,2) \\ \frac{(3-t)^{2}}{2} & t \in[2,3) \\ 0 & t \notin[0,3) .\end{cases}
$$

This generator is suitable for computations and it satisfies the Strang-Fix conditions of order $r=3$. As $\operatorname{supp} \varphi=[0,3]$ we could take $p=3$, i.e., the sampling period $T_{\mathrm{s}}=3 / 4$. The corresponding $4 \times 3$ matrix $H(z)$ is

$$
H(z)=\frac{1}{32}\left(\begin{array}{ccc}
0 & 16 & 16 \\
9 & 22 & 1 \\
24 & 4 & 0 \\
9 & 0 & 0
\end{array}\right)+\frac{1}{32}\left(\begin{array}{ccc}
0 & 0 & 0 \\
0 & 0 & 0 \\
0 & 0 & 4 \\
0 & 1 & 22
\end{array}\right) z
$$

The polynomial left inverse $G(z)$ above computed (19) gives us the reconstruction functions [see (20)]

$$
\begin{aligned}
S_{0}^{G}(t)= & \frac{1}{54} N_{3}(t)-\frac{13}{126} N_{3}(t+1)+\frac{265}{126} N_{3}(t+2) \\
& +\frac{1}{54} N_{3}(t+3)-\frac{1}{126} N_{3}(t+4) \\
& +\frac{1}{126} N_{3}(t+5) \\
S_{1}^{G}(t)= & \frac{-8}{27} N_{3}(t)+\frac{104}{63} N_{3}(t+1)-\frac{104}{63} N_{3}(t+2) \\
S_{2}^{G}(t)= & \frac{14}{9} N_{3}(t)-\frac{2}{3} N_{3}(t+1)+\frac{2}{3} N_{3}(t+2) \\
S_{3}^{G}(t)= & -\frac{8}{27} N_{3}(t)+\frac{8}{63} N_{3}(t+1)-\frac{8}{63} N_{3}(t+2) .
\end{aligned}
$$

From Theorem 1, we obtain that, any $f \in V_{N_{3}}^{\infty}$ can be expanded as

$$
f(t)=\sum_{j=0}^{3} \sum_{n \in \mathbb{Z}} f\left(3 n+j \frac{3}{4}\right) S_{j}^{G}(t-3 n), \quad t \in \mathbb{R} .
$$

Moreover, from Theorem 2 we deduce that, for any $f \in W_{\infty}^{3}(\mathbb{R})$ we have

$$
\begin{array}{r}
\left\|f(t)-\sum_{j=0}^{3} \sum_{n \in \mathbb{Z}} f\left(j \frac{3}{4} h+3 n h\right) S_{j}^{G}\left(\frac{t}{h}-3 n\right)\right\|_{\infty} \\
\leq K\left\|D^{3} f\right\|_{\infty} h^{3}
\end{array}
$$

where $K$ is a constant independent of $f$ and $h$. In other words, the sampling formula (21) allow us to recover any function in $V_{N_{3}}^{\infty}$ from its samples taken at the points $\{(3 / 4) n\}_{n \in \mathbb{Z}}$, and to approximate any function in $W_{\infty}^{3}(\mathbb{R}) \cap C(\mathbb{R})$ with order 3 . The reconstruction functions $S_{1}^{G}, S_{2}^{G}, S_{3}^{G}$ are supported in the interval $[-2,3]$, whilst $S_{0}^{G}$ is supported in the interval $[-5,3]$.

\section{A Comparison With the Orthogonal Projector}

Blue and Unser gave in [2] an expression for the $L^{2}$-error valid to an ample set of approximation schemes of functions $f \in W_{2}^{r}(\mathbb{R}):=\left\{f: \int\left(1+w^{2}\right)^{r}|\widehat{f}(w)|^{2} d w<\infty\right\}$. In our approximation scheme (14), it reads (see the Appendix ):

$$
\begin{aligned}
\left\|f(t)-\sum_{j=0}^{q-1} \sum_{n \in \mathbb{Z}} f\left(j T_{\mathrm{s}} h+p n h\right) S_{j}^{G}\left(\frac{t}{h}-p n\right)\right\|_{2}^{2} \\
=\int|\widehat{f}(w)|^{2} E(h w) d w+O\left(h^{r}\right) .
\end{aligned}
$$


The kernel $E(w)$ is given by

$$
\begin{aligned}
E(w)= & \frac{1}{p^{2}}\left|p-\rho_{p}^{\top}(z) G\left(z^{p}\right) \rho_{q}\left(z^{T_{\mathrm{s}}}\right) \overline{\hat{\varphi}(w)}\right|^{2} \\
& +\frac{1}{p^{2}} \sum_{n \neq 0}\left|\rho_{p}^{\top}\left(z e^{-2 \pi i n / p}\right) G\left(z^{p}\right) \rho_{q}\left(z^{T_{\mathrm{s}}}\right) \overline{\hat{\varphi}\left(w+\frac{n}{p}\right)}\right|^{2}
\end{aligned}
$$

where $\rho_{l}(s)=\left[1, s, \ldots, s^{l-1}\right]^{\top}, l=p, q$ and $z=e^{-2 \pi i w}$. Moreover, $\int|\widehat{f}(w)|^{2} E(h w) d w$ gives exactly the average approximation error (see [2, Theorem 2]). In particular, when the sampling period is $T_{\mathrm{s}}=1 / q$, i.e., $p=1$, the kernel can be expressed as

$$
E(w)=E_{\min }(w)+\widehat{a}_{\varphi}(w)\left|\widehat{\varphi}_{d}(w)-G(z) \rho_{q}\left(z^{T_{\mathrm{s}}}\right)\right|^{2}
$$

where $\widehat{a}_{\varphi}(w)=\sum|\widehat{\varphi}(w+n)|^{2}, \varphi_{d}(w)$ is the dual function (11), and $E_{\min }(w)=1-|\widehat{\varphi}(w)|^{2} / \widehat{a}_{\varphi}(w)$ is the kernel corresponding to the orthogonal projector (10) (see [3]). This expression for the error provides a criterion in order to get an approximation scheme close to the orthogonal projector. Namely, in cases where most of the spectral energy is concentrated in a neighborhood of $w=0$, we could try to find a matrix $G(z)$ (satisfying $G(z) H(z)=1$ ) such that

$$
\widehat{\varphi}_{d}(w)-G(z) \rho_{q}\left(z^{T_{\mathrm{s}}}\right)=O\left(w^{L}\right)
$$

for a big enough $L$, and having at the same time reconstruction functions $S_{j}^{G}$ with small support. Notice that this technique has been borrowed from [3] (see also [6] and [7]) where quasi-interpolating schemes are used.

For instance, we consider the generator $\varphi(t)=N_{3}^{c}(t)=$ $N_{3}(t+3 / 2)$, i.e., the centered quadratic B-spline, and sampling period $T_{\mathrm{s}}=1 / 2$. In this case, the matrix $H(z)$ reads: $H(z)=$ $[z / 8+3 / 4+(1 / 8) z, z / 2+1 / 2]^{\top}$; its polynomial left inverse matrices $G(z):=\left[G_{0,0}(z), G_{0,1}(z)\right]$ are described by

$$
\begin{aligned}
& G_{0,0}(z)=2+\left(\frac{z}{2}+\frac{1}{2}\right) p(z) \\
& G_{0,1}(z)=-\frac{1}{2}-\frac{1}{2 z}-\left(\frac{z}{8}+\frac{3}{4}+\frac{1}{8 z}\right) p(z)
\end{aligned}
$$

where $p(z)$ is any Laurent polynomial. Having in mind that

$$
\widehat{\varphi}_{d}(w)=\frac{\sin ^{3}(\pi w)}{\pi^{3} w^{3}\left[1-\sin ^{2}(\pi w)+\left(\frac{2}{15}\right) \sin ^{4}(\pi w)\right]}
$$

(see [3]) we obtain that, for any choice of $p(z)$, estimation (25) for $L=4$ holds. It is one order more than the expected one (we have approximation order 3 ) which is explained because, in this example, $\widehat{\varphi}_{d}(w)-G(z) \rho_{q}\left(z^{T_{\mathrm{s}}}\right)=\xi(w)+\eta(w) p(z) e^{-i \pi w}$ where $\xi, \eta$ are even functions. The choice $p(z) \equiv 0$ gives the reconstruction functions with small support, $S_{0}^{G}(t)=2 N_{3}^{c}(t)$ and $S_{1}^{G}(t)=(-1 / 2)\left[N_{3}^{c}(t)+N_{3}^{c}(t-1)\right]$, and the associated approximation scheme reads

$$
\sum_{n \in \mathbb{Z}}\left[f(n h) S_{0}^{G}\left(\frac{t}{h}-n\right)+f\left(\frac{h}{2}+n h\right) S_{1}^{G}\left(\frac{t}{h}-n\right)\right] .
$$

The choice $p(z) \equiv-22 / 15$ gives $L=5$, being the reconstruction functions, $S_{0}^{G}(t)=(19 / 15) N_{3}^{c}(t)-(11 / 15) N_{3}^{c}(t+1)$ and $S_{1}^{G}(t)=(3 / 5) N_{3}^{c}(t)-(19 / 60) N_{3}^{c}(t-1)+(11 / 60) N_{3}^{c}(t+1)$. The choice $p(z)=(-11 / 15)\left(1+z^{-1}\right)$ gives $L=6$ with reconstruction functions with a bigger support.

Finally, let us give a numerical simulation showing the behavior of the studied approximation formulas. We apply the above formulas to approximate the Gaussian function $f(t)=$ $e^{-t^{2}}$ from 80 samples taken in the interval $[-4,4]$ with sampling period 0.1 . The formula obtained for the choice $p(z) \equiv 0$, which has the smallest support, gives an $L^{2}$-norm error equal to $2.9 \times 10^{-4}$. The formula obtained for the choice $p(z) \equiv$ $-22 / 25$, which is closer to the orthogonal projector, gives an error equal to $2.2 \times 10^{-4}$. The formula (22) obtained in the previous section gives an error equal to $8.5 \times 10^{-5}$. This error is smaller due to less oversampling being introduced $\left(T_{\mathrm{s}}=3 / 4\right)$, but its bigger support implies more computations than in the first one. Classical quadratic interpolation formula gives an error equal to $2.5 \times 10^{-5}$. This error is the smallest one (oversampling is not used here), but it implies more computations than in the previous cases. For the last estimation we have used the approximation $\sqrt{2} \sum_{n=-9}^{8}(2 \sqrt{2}-3)^{|n+1|} N_{3}(t-n)$ for the quadratic interpolating spline. Other numerical experiments show a similar behavior.

\section{APPENDIX}

In this Appendix, we include the technical proofs of the results in Sections II and III.

Proof of Lemma 1: Notice first that the pointwise multiplication is a closed operation in $\mathcal{A}$. Moreover, the Wiener's lemma (see, e.g., [13]) establishes that if $f \in \mathcal{A}$ and $f(z) \neq 0$ on $|z|=1$, then the function $1 / f$ is also in $\mathcal{A}$. Notice also that the entries of the matrix $H(z)$ belong to $\mathcal{A}$, since we have assumed that $\varphi \in W\left(L^{\infty}, \ell^{1}\right)$. If $H(z)$ has a left inverse on $|z|=1$ then $H(z)$ has full rank on $|z|=1$.

Reciprocally, if $H(z)$ has full rank on $|z|=1$ or, equivalently, if $\operatorname{det} H^{*}(z) H(z) \neq 0$ on $|z|=1$, then the pseudo-inverse $H^{\dagger}(z):=\left[H^{*}(z) H(z)\right]^{-1} H^{*}(z)$ does exist on $|z|=1$. It is a left inverse of $H(z)$ and its entries belong to $\mathcal{A}$ by the Wiener's lemma.

It can be checked that $H^{\dagger}(z)+U(z)\left[\rrbracket_{q}-H(z) H^{\dagger}(z)\right]$, where $U(z)$ is a $p \times q$ matrix with entries in $\mathcal{A}$, is a left inverse of $H(z)$ with entries in $\mathcal{A}$. Moreover, if $G(z)$ is a left inverse of $H(z)$ with entries in $\mathcal{A}$, it can be expressed as $G(z)=$ $H^{\dagger}(z)+U(z)\left[\rrbracket_{q}-H(z) H^{\dagger}(z)\right]$ by taking $U(z)=G(z)$.

In proving Theorem 1 and Theorem 2, the sampling operator $\Gamma^{G}$, formally defined as

$$
\left(\Gamma^{G} f\right)(t):=\sum_{j=0}^{q-1} \sum_{n \in \mathbb{Z}} f\left(j T_{\mathrm{s}}+p n\right) S_{j}^{G}(t-p n), \quad t \in \mathbb{R} .
$$

will play an important role; defining $\Gamma^{G}$ in $C_{b}(\mathbb{R})$, the space of continuous and bounded functions endowed with the $L^{\infty}$-norm, the following result holds.

Lemma 2: Under the hypotheses of Theorem 1, the sampling operator $\Gamma^{G}$ is a well-defined bounded operator from $C_{b}(\mathbb{R})$ to $L^{\infty}(\mathbb{R})$. 
Proof: For $j=0,1, \ldots, q-1$, we have

$$
\begin{aligned}
& \sup _{t \in[0,1)} \sum_{n \in \mathbb{Z}}\left|S_{j}^{G}(t-n)\right| \\
& \quad=\sup _{t \in[0,1)} \sum_{n \in \mathbb{Z}}\left|\sum_{m \in \mathbb{Z}} g_{j}[m] \varphi(t-n-m)\right| \\
& \quad \leq \sup _{t \in[0,1)} \sum_{m \in \mathbb{Z}}\left|g_{j}[m]\right| \sum_{n \in \mathbb{Z}}|\varphi(t-n-m)| \\
& \quad \leq\left\|g_{j}\right\|_{1} \sup _{t \in[0,1)} \sum_{n \in \mathbb{Z}}|\varphi(t-n)| .
\end{aligned}
$$

Thus, for every $f \in C_{b}(\mathbb{R})$, the function $\Gamma^{G} f$ belongs to $L^{\infty}(\mathbb{R})$, and the sampling operator $\Gamma^{G}$ is well-defined; notice also that the functions $S_{j}^{G}, j=0,1, \ldots, q-1$, belong to the Wiener space $W\left(L^{\infty}, \ell^{1}\right)$. Moreover

$$
\begin{aligned}
\left|\left(\Gamma^{G} f\right)(t)\right| & =\left|\sum_{j=0}^{q-1} \sum_{n \in \mathbb{Z}} f\left(j T_{\mathrm{s}}+p n\right) S_{j}^{G}(t-p n)\right| \\
& \leq\left(\sum_{j=0}^{q-1} \sup _{t \in[0,1)} \sum_{n \in \mathbb{Z}}\left|S_{j}^{G}(t-n)\right|\right)\|f\|_{\infty} .
\end{aligned}
$$

As a consequence, we obtain that $\left\|\Gamma^{G} f\right\|_{\infty} \leq K\|f\|_{\infty}$ for some constant $K$ independent of $f$, which proves the lemma.

Proof of Theorem 1: First, we prove that the sampling formula (9) is satisfied for any function $f$ in $\operatorname{span}\{\varphi(\cdot-n)\}_{n \in \mathbb{Z}}$, i.e., for any $f$ of the form $f(t)=\sum_{n \in \mathbb{Z}} a[n] \varphi(t-n)$ where $\{a[n]\}$ is a finite sequence. Notice that the z-transform establishes a biyection between the space $\ell^{1}(\mathbb{Z})$ and the class $\mathcal{A}$, where the convolution operator in $\ell^{1}(\mathbb{Z})$ corresponds to the multiplication of functions in $\mathcal{A}$.

From (5), and using that $G(z)$ is a left inverse to $H(z)$, we obtain

$$
\begin{aligned}
G(z)\left[C_{0}(z) C_{1}(z) \cdots C_{q-1}(z)\right]^{\top} & \\
= & {\left[A_{0}(z) A_{1}(z) \cdots A_{p-1}(z)\right]^{\top} . }
\end{aligned}
$$

Observe that $G_{k, j}(z)=\sum_{n \in \mathbb{Z}} g_{j}[-k+p n] z^{-n}$ is the z-transform of the sequence $\left\{g_{k, j}[n]=g_{j}[-k+p n]\right\}_{n \in \mathbb{Z}}$. Then, for $k=0,1, \ldots, p-1$, we have

$$
\begin{aligned}
a[-k+n p] & =a_{k}[n]=\sum_{j=0}^{q-1}\left(g_{k, j} * c_{j}\right)[n] \\
& =\sum_{j=0}^{q-1} \sum_{m \in \mathbb{Z}} c_{j}[m] g_{j}[-k+p n-p m], \quad n \in \mathbb{Z} .
\end{aligned}
$$

Hence

$$
\begin{aligned}
a[n] & =\sum_{j=0}^{q-1} \sum_{m \in \mathbb{Z}} c_{j}[m] g_{j}[n-p m] \\
& =\sum_{j=0}^{q-1} \sum_{m \in \mathbb{Z}} f\left(p m+j T_{\mathrm{S}}\right) g_{j}[n-p m], \quad n \in \mathbb{Z} .
\end{aligned}
$$

This formula allows us to recover the finite sequence $\{a[n]\}$ from the samples. As a consequence, the function $f(t)=\sum_{n \in \mathbb{Z}} a[n] \varphi(t-n)$ can be recovered in the following way:

$$
\begin{aligned}
f(t)= & \sum_{n \in \mathbb{Z}} a[n] \varphi(t-n) \\
= & \sum_{n \in \mathbb{Z}} \sum_{j=0}^{q-1} \sum_{m \in \mathbb{Z}} f\left(p m+j T_{\mathrm{s}}\right) \\
& \times g_{j}[n-p m] \varphi(t-n) \\
= & \sum_{j=0}^{q-1} \sum_{m \in \mathbb{Z}} f\left(p m+j T_{\mathrm{s}}\right) \sum_{n \in \mathbb{Z}} g_{j}[n-p m] \varphi(t-n) \\
= & \sum_{j=0}^{q-1} \sum_{m \in \mathbb{Z}} f\left(p m+j T_{\mathrm{s}}\right) \sum_{n \in \mathbb{Z}} g_{j}[n] \varphi(t-n-p m) \\
= & \sum_{j=0}^{q-1} \sum_{m \in \mathbb{Z}} f\left(p m+j T_{\mathrm{s}}\right) S_{j}^{G}(t-p m) .
\end{aligned}
$$

Next we prove that the sampling formula (9) holds for every function $f(t)=\sum_{n \in \mathbb{Z}} a[n] \varphi(t-n)$ in $V_{\varphi}^{\infty}$. The sequence of functions $f_{M}(t):=\sum_{|n| \leq M} a[n] \varphi(t-n)$ in $V_{\varphi}^{\infty}$ converges to $f$ uniformly on $\mathbb{R}$ because

$$
\sum_{|n|>M}|a[n] \varphi(t-n)| \leq \sup _{|n|>M}|a[n]| \sup _{t \in[0,1)} \sum_{n \in \mathbb{Z}}|\varphi(t-n)|
$$

$\lim _{|n| \rightarrow \infty} a[n]=0$ and $\varphi \in W\left(L^{\infty}, \ell^{1}\right)$. Since $f_{M} \in \operatorname{span}\{\varphi(\cdot-n)\}_{n \in \mathbb{Z}}$ we have that $\Gamma^{G} f_{M}=f_{M}$. From Lemma $2, \Gamma^{G}$ is a bounded operator in $C_{b}(\mathbb{R})$. Denoting by $\left\|\Gamma^{G}\right\|$ its norm, for every $M \in \mathbb{N}$, we get

$$
\begin{aligned}
\left\|f-\Gamma^{G} f\right\|_{\infty} & \leq\left\|f-f_{M}\right\|_{\infty}+\left\|\Gamma^{G} f_{M}-\Gamma^{G} f\right\|_{\infty} \\
& \leq\left\|f-f_{M}\right\|_{\infty}+\left\|\Gamma^{G}\right\|\left\|f-f_{M}\right\|_{\infty}
\end{aligned}
$$

from which we deduce that $f=\Gamma^{G} f$ for each $f \in V_{\varphi}^{\infty}$ or, in other words, the sampling formula (9) holds in $V_{\varphi}^{\infty}$.

It remains to prove the absolute and uniform convergence of the series in (9). As $\lim _{|t| \mapsto \infty} \varphi(t)=0$, we have that $\lim _{|t| \rightarrow \infty} f_{M}(t)=0$. Since $f_{M}$ converges uniformly to $f$ on $\mathbb{R}$ we have that $\lim _{|n| \rightarrow \infty} f\left(n T_{\mathrm{s}}\right)=0$. Using (27), we obtain

$$
\begin{aligned}
\sum_{|n|>N} \mid f\left(j T_{\mathrm{s}}\right. & +p n) S_{j}^{G}(t-p n) \mid \\
& \leq \sup _{|n|>N}\left|f\left(j T_{\mathrm{s}}+p n\right)\right|\left\|g_{j}\right\|_{1} \sup _{t \in[0,1)} \sum_{n \in \mathbb{Z}}|\varphi(t-n)|
\end{aligned}
$$

for $t \in \mathbb{R}, j=0,1, \ldots, q-1$ and $N \in \mathbb{N}$. From this inequality, using that $\lim _{|n| \mapsto \infty} f\left(T_{\mathrm{s}} n\right)=0$ and that $\varphi \in W\left(L^{\infty}, \ell^{1}\right)$ we deduce that the convergence of the series in (9) is absolute and also uniform on $\mathbb{R}$.

Proof of Theorem 2: Let $f \in W_{\infty}^{r}(\mathbb{R}) \cap C(\mathbb{R})$; the operator $P$ defined in (10) projects $L^{\infty}(\mathbb{R})$ onto $V_{\varphi}^{\infty}$. In particular, $P \sigma_{1 / h} f \in V_{\varphi}^{\infty}$. Hence, using Theorem 1 we obtain $\Gamma^{G} P \sigma_{1 / h} f=P \sigma_{1 / h} f$, where $\Gamma^{G}$ is the sampling operator defined in (26) and, consequently

$$
\sigma_{h} P \sigma_{1 / h} f=\left(\sigma_{h} \Gamma^{G} \sigma_{1 / h}\right)\left(\sigma_{h} P \sigma_{1 / h}\right) f .
$$


Using this equality, we obtain

$$
\begin{aligned}
\| f(t) & -\sum_{j=0}^{q-1} \sum_{n \in \mathbb{Z}} f\left(j T_{\mathrm{s}} h+p n h\right) S_{j}^{G}\left(\frac{t}{h}-p n\right) \|_{\infty} \\
= & \left\|f-\sigma_{h} \Gamma^{G} \sigma_{1 / h} f\right\|_{\infty} \\
\leq & \left\|f-\sigma_{h} P \sigma_{1 / h} f\right\|_{\infty} \\
& +\left\|\sigma_{h} P \sigma_{1 / h} f-\sigma_{h} \Gamma^{G} \sigma_{1 / h} f\right\|_{\infty} \\
= & \left\|f-\sigma_{h} P \sigma_{1 / h} f\right\|_{\infty} \\
& +\left\|\sigma_{h} \Gamma^{G} \sigma_{1 / h}\left(\sigma_{h} P \sigma_{1 / h} f-f\right)\right\|_{\infty} \\
\leq & \left\|f-\sigma_{h} P \sigma_{1 / h} f\right\|_{\infty} \\
& +\left\|\sigma_{h} \Gamma^{G} \sigma_{1 / h}\right\|\left\|\left(\sigma_{h} P \sigma_{1 / h} f-f\right)\right\|_{\infty} \\
= & \left(1+\left\|\Gamma^{G}\right\|\right)\left\|f-\sigma_{h} P \sigma_{1 / h} f\right\|_{\infty}
\end{aligned}
$$

where $\left\|\Gamma^{G}\right\|$ and $\left\|\sigma_{h} \Gamma^{G} \sigma_{1 / h}\right\|$ denote the norms of the operators $\Gamma^{G}, \sigma_{h} \Gamma^{G} \sigma_{1 / h}: C_{b}(\mathbb{R}) \longrightarrow L^{\infty}(\mathbb{R})$ which coincide (see Lemma 2). Finally, the theorem follows from inequality (13).

Proof of the Error Formula (23): It can easily checked that

$$
\begin{aligned}
\sum_{j=0}^{q-1} \sum_{n \in \mathbb{Z}} f\left(j T_{\mathrm{s}} h\right. & +p n h) S_{j}^{G}\left(\frac{t}{h}-p n\right) \\
= & \sum_{n \in \mathbb{Z}} \int f(\tau) \widetilde{\Phi}^{\top}\left(\frac{\tau}{h}-p n\right) \Phi\left(\frac{t}{h}-p n\right) \frac{d \tau}{h}
\end{aligned}
$$

where $\Phi(t)=[\varphi(t), \varphi(t+1), \ldots, \varphi(t+p-1)]^{\top}$, and $\widetilde{\Phi}=$ $\left[\widetilde{\phi}_{0}, \ldots, \widetilde{\phi}_{p-1}\right]^{\top}$ is given by

$\widetilde{\phi}_{k}(t)=\sum_{j=0}^{q-1} \sum_{n \in \mathbb{Z}} g_{j}[p n-k] \delta\left(t-j T_{\mathrm{s}}+p n\right)$,

$$
k=0,1, \ldots, p-1
$$

where $\delta$ denotes the Dirac delta. Hence, by applying [2, Theorem 1], the error formula (23) is obtained. By using that $G_{k, j}(z)=\sum_{m \in \mathbb{Z}} g_{j}[-k+p m] z^{-m}$ we obtain

$$
\begin{aligned}
\widehat{\widetilde{\phi}}_{k}(w) & :=\sum_{j=0}^{q-1} \sum_{n \in \mathbb{Z}} g_{j}[p n-k] e^{-2 \pi i\left(j T_{\mathrm{s}}-p n\right) w} \\
& =\sum_{j=0}^{q-1} e^{-2 \pi i j T_{\mathrm{s}} w} G_{k, j}\left(e^{-2 \pi i p w}\right)
\end{aligned}
$$

and, as a consequence, $\widehat{\widetilde{\Phi}}(w)=G\left(e^{-2 \pi i p w}\right) \rho_{q}\left(e^{-2 \pi i T_{\mathrm{s}} w}\right)$. Hence, as $\widehat{\Phi}(w)=\rho_{p}\left(e^{2 \pi i w}\right) \widehat{\varphi}(w)$, we obtain the expression of the kernel (24).

\section{ACKNOWLEDGMENT}

The authors wish to thank the referees for their valuable and constructive comments. They have helped to improve both the contain and the presentation of the paper.

\section{REFERENCES}

[1] A. Aldroubi and M. Unser, "Sampling procedures in function spaces and asymptotic equivalence with Shannon's sampling theory," Numer. Funct. Anal. Optimiz., vol. 15, no. 1, pp. 1-21, 1994.

[2] T. Blu and M. Unser, "Approximation error for quasi-interpolators and (multi-)Wavelet expansions," Appl. Comput. Harmon. Anal., vol. 6, no. 2, pp. 219-251, 1999.
[3] T. Blu and M. Unser, "Quantitative Fourier analysis of approximation techniques: Part I-interpolators and projectors, and Part II-wavelets," IEEE Trans. Signal Process., vol. 47, no. 10, pp. 2783-2806, Oct. 1999.

[4] C. de Boor, R. DeVore, and A. Ron, "Approximation from shift-invariant subspaces in $L^{2}\left(\mathbb{R}^{d}\right)$, , Trans. Amer. Math. Soc., vol. 341, pp. 787-806, 1994.

[5] O. Christensen, An Introduction to Frames and Riesz Bases. Boston, MA: Birkhäuser, 2003.

[6] L. Condat, T. Blu, and M. Unser, "Beyond interpolation: Optimal reconstruction by quasi-interpolation," in Proc. IEEE Int. Conf. Image Processing Trans., 2005, vol. 1, pp. 33-36.

[7] L. Condat and D. Van De Ville, "Quasi-interpolating spline models for hexagonally-sampled data," IEEE Trans. Signal Process., vol. 16, no. 5, pp. 1195-1206, May 2007.

[8] I. Djokovic and P. P. Vaidyanathan, "Generalized sampling theorems in multiresolution subspaces," IEEE Trans. Signal Process., vol. 45, no. 3, pp. 583-599, Mar. 1997.

[9] A. Fischer, "Multiresolution analysis and multivariate approximation of smooth signals in $C_{b}\left(\mathbb{R}^{d}\right)$," J. Fourier Anal. Appl., vol. 2, pp. $162-180,1995$

[10] A. G. García and G. Pérez-Villalón, "Approximation from shift-invariant spaces by generalized sampling formulas," Appl. Comput. Harmon. Anal., vol. 24, no. 1, pp. 58-69, 2008.

[11] J. J. Lei, R. Q. Jia, and E. W. Cheney, "Approximation from shiftinvariant spaces by integral operators," SIAM J. Math. Anal., vol. 28 , no. 2, pp. 481-498, 1997.

[12] R. Rajagopal and L. C. Poter, "Multivariate MIMO FIR inverses," IEEE Trans. Image Process., vol. 12, no. 4, pp. 458-465, 2003.

[13] W. Rudin, Functional Analysis. New York: McGraw-Hill, 1973.

[14] M. Unser and I. Daubechies, "On the approximation power of convolution-based least squares versus interpolation," IEEE Trans. Signal Process, vol. 45, no. 7, pp. 1697-1711, Jul. 1997.

[15] M. Unser and J. Zerubia, "A generalized sampling theory without bandlimiting constraints," IEEE Trans. Circuits Syst., vol. 45, no. 8, pp. 959-969, Aug. 1998

[16] M. Unser, "Sampling-50 years after Shannon," Proc. IEEE, vol. 88, pp. $569-587,2000$.

[17] G. G. Walter, "A sampling theorem for wavelet subspaces," IEEE Trans. Inf. Theory, vol. 38, no. 2, pp. 881-884, Mar. 1992.

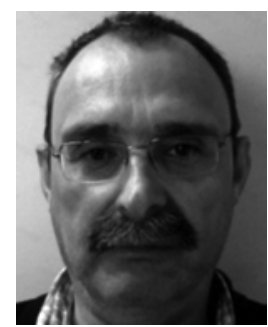

A. G. García was born in Valladolid, Spain, in 1957. He received the M.Sc. degree in mathematics and the Ph.D. in mathematics, both from the University of Valladolid, Spain, in 1979 and 1983, respectively.

After teaching mathematics in a high school, in 1991 he joined the Department of Mathematics of the University Carlos III de Madrid, Spain, as an Associate Professor. His research interests include all the mathematical topics related to wavelets and sampling theory.

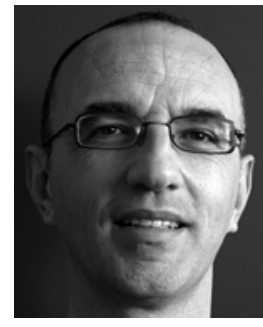

M. A. Hernández-Medina was born in Getafe, Spain, in 1964. He received the M.Sc. degree in mathematics from the Universidad Complutense de Madrid, Spain, in 1987 and the Ph.D. degree in mathematics from the Universidad Politécnica de Madrid, Spain, in 1997.

Since 2000, he has been an Associate Professor at the E.T.S.I.T. of the Universidad Politécnica de Madrid. His research interest include sampling theory, wavelets, harmonic analysis, and approximation theory.

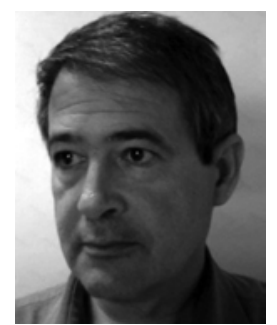

G. Pérez-Villalón was born in Teruel, Spain, in 1960. He received the M.Sc. degree in mathematics from the University Complutense of Madrid, Spain, in 1983 and the Ph.D. in mathematics from the University Carlos III de Madrid, Spain, in 2005.

$\mathrm{He}$ is currently Associate Professor in the Department of Mathematics of the EUITT in the Madrid Polytechnic University, Spain. His research interests include wavelets, interpolation, approximation, and sampling theory. 